\title{
2-D COMPLEX WAVELET TRANSFORMS FOR IDENTIFICATION OF WAVE-LIKE FEATURES IN GEOPHYSICAL DATA
}

\author{
Piyush Shanker A, and Ravi S. Nanjundiah \\ Center for Atmospheric and Oceanic Sciences, \\ Indian Institute of Science, \\ Bangalore, India. \\ e-mail: piyush@caos.iisc.ernet.in, \\ ravi@caos.iisc.ernet.in (Corresponding author)
}

\begin{abstract}
The power spectrum of a two-dimensional (2-D) space-time data set is computed using a 2-D extension of the Continuous Morlet Wavelet Transform. By means of this method, the power specträ in 4-D space (spatial scale-time periodspace-time) can be estimated. This method is very useful in analyzing waves as it enables us to track the variation of frequency and amplitude of a wave with time and space. The method has an added advantage that we can obtain the predominant spatial scales over a particular region, the temporal scales associated with them and their time of occurrence. An example of it's application to study the features of poleward propagation of monsoons over the Indian longitudes and the pacific is given. This method can be extended for application to multi-dimensional datasets.
\end{abstract}

\section{INTRODUCTION}

Conventional 2-D spectral analysis methods utilizing a simple Fourier transform or the Maximum Entropy Method [1], decompose a signal into a two-dimensional wavenumberfrequency space. These methods yield no information regarding the time evolution or the regional variation of the spectral properties.

Continuous wavelet transforms have been used as an effective data analysis tool for one-dimensional data series. The greatest advantage of using wavelet transforms on a time series is the simultaneous localization in the frequency and time domains. [2] laid down the practical mathematical framework for applying wavelet analysis to a one-dimensional time series.

Methods combining the wavelet analysis with the traditional Fourier analysis like the one proposed in [3] also suffer from a similar setback. They either provide wavenumber-

\footnotetext{
The work presented here was partially supported under the CSIR. NMITLI project on Meso-scale modelling for Monsoon related predictions.
}

frequency information with localization in space or in time. We cannot obtain localization in both time and spatial domains simultaneously using such techniques. The 2-D extension of the continuous wavelet transform [4] is capable of providing this information.

\section{MATHEMATICAL FORMULATION}

The complex Morlet Wavelet was used for the two-dimensional analysis. The Morlet mother wavelet $\left(\psi_{0}(\eta)\right)$ is given by the equation:-

$$
\psi_{0}(\eta)=\pi^{-0.25} e^{\left(j \omega_{0} \eta\right)} e^{\left(-\eta^{2} / 2\right)} \quad\left(\omega_{0}>5.0\right)
$$

The Morlet wavelet transform of a signal at a particular scale is given by the convolution of the signal with a scaled version of the mother wavelet. The term "scale" has been synonymously used for the "Fourier wavelength" throughout. The scaled version is normalized to have unit energy. The value $\omega_{0}=6.0$ has been used throughout the analysis. The Morlet wavelet is ideal for analysis of geophysical data because :

1. It can be easily visualized as a wave because of it's oscillatory nature and has a neat closed form expression.

2. It captures both the maxima and minima similarly, unlike real wavelets, enabling better comparison of the power associated with various spectral components.

3. The phase information obtained could be used for velocity studies.

4. The one-sided nature of it's Fourier transform enables us to use the time-reversed representation of the wavelet to capture negative-frequencies. 


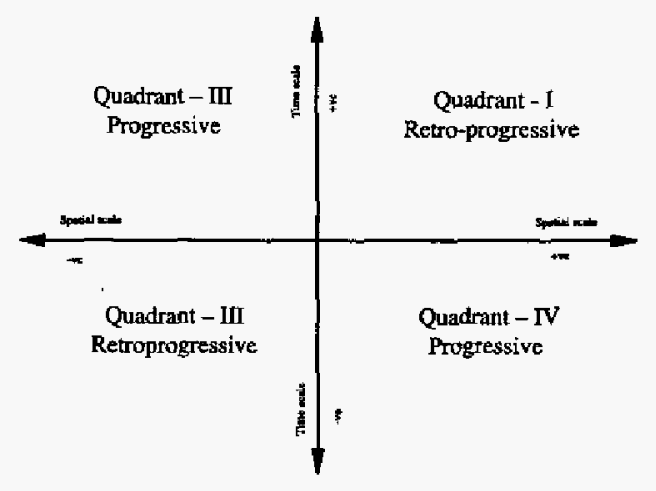

Fig. 1. Figure depicting the method of choosing scales for obtain specific spectral details of progressive and retroprogressive waves.

The convolution with data is carried out in the frequency domain to save on the computation and the Fourier transform of the scaled wavelet in the discrete time domain would be given by

$$
\hat{\psi}_{s}\left(s \omega_{k}\right)=\sqrt{\frac{2 \pi|s|}{\delta t}} \pi^{-0.25} e^{\frac{-\left(\frac{2 \pi s k}{N \delta !}-\omega_{0}\right)^{2}}{2}} u\left(s \omega_{k}\right)
$$

where $u\left(s \omega_{k}\right)$ represents the step function.

Let $f(x, t)$ represent the two-dimensional data set with " $x$ " representing the spatial domain and " $t$ " representing the time domain. The one-dimensional wavelet transform was computed over the spatial domain at each time instant. Let the $y\left(s_{x}, x, t\right)$ represent the resultant three-dimensional transform. Here " $s_{x}$ " represents the spatial scale under consideration.

$$
y\left(s_{x}, x, t\right)=f(x, t) * \frac{1}{\sqrt{\left|s_{x}\right|}} \psi_{0}\left(\frac{x}{s_{x}}\right)
$$

The 4-D spectra $\left(w\left(s_{x}, s_{t}, x, t\right)\right)$ was obtained after applying the wavelet transform over the time domain as well. The corresponding mathematical expression is given by:-

$$
w\left(s_{x}, s_{t}, x, t\right)=y\left(s_{x}, x, t,\right) * \frac{1}{\sqrt{\left|s_{t}\right|}} \psi_{0}\left(\frac{t}{s_{t}}\right)
$$

The progressive and retro-progressive spectral components can be specifically identified by assigning appropriate values to the scale parameters, " $s_{x}$ " and " $s_{t} "$. Fig. 1 shows the importance of the sign of the scales chosen for analysis.

\subsection{Cone of influence}

All convolutions are performed in the Fourier domain to speed up the computations. Hence, the data as well as the wavelet are assumed to be periodic. The computed transform suffers from edge defects and the co-efficients at the edges of the series are corrupted by the periodic wrapping. The maximum reliable period for a $1-\mathrm{D}$ transform at a given position " $n$ " of an " $N$ " length series is given by ([2]):

$$
\operatorname{Maxperiod}(n)=\bmod \left(n, \frac{N}{2}\right) * \frac{1}{\sqrt{2}}
$$

As a result, we often need to use data series of longer length to obtain reliable results over the regions of interest.

\section{METHODS}

The 4-D spectra can be manipulated in different ways to obtain characteristic information about the geophysical phenomenon represented by the data. A few analysis methods are briefly explained.

\subsection{Identification of dominant Spatial Scales}

This method involves the identification of dominant spatial scales at different points in space. The 4-D spectra is averaged over a time period of interest and a range of time scales like synoptic (5-10 days) and intra-seasonal (10-70 days) to obtain spatial scale v/s space plots.

$$
\hat{P}\left(s_{x}, x\right)=\sum_{s_{i 1}}^{s_{12}} \sum_{t_{1}}^{t_{2}}\left|w\left(s_{x}, s_{i}, x, t\right)\right|^{2}
$$

where $s_{t 1}$ to $s_{t 2}$ represents the range of time scales of interest and $t_{1}$ to $t_{2}$ represents the time interval of interest. These plots are also called as time-averaged scalograms.

\subsection{Time evolution of the dominant spatial scales}

The 4-D spectra is averaged over a region of interest in the spatial domain and over a range of pertinent spatial scales to observe the time-evolution of the geophysical phenomenon. Mathematically, it can be defined as

$$
\hat{Q}\left(s_{t}, t\right)=\sum_{s_{x 1}}^{s_{x 2} 2} \sum_{x_{1}}^{x_{2}}\left|w\left(s_{x}, s_{t}, x, t\right)\right|^{2}
$$

\subsection{Correlation between different phenomenon}

The 4-D spectra can also be used to identify characteristic relations between two phenomenon. This method also enables us to identify the relations between the different spatial and time scales characterizing both the phenomenon.

$\hat{C}_{w_{1}, w_{2}}\left(s_{x}^{1}, s_{t}^{1}, s_{x}^{2}, s_{t}^{2}, x, t\right)=\left|w_{1}\left(s_{x}^{1}, s_{t}^{1}, x, t\right) \cdot w_{2}^{*}\left(s_{x}^{2}, s_{t}^{2}, x, t\right)\right|$ 
The function $C_{w_{1}, w_{2}}$ can be averaged over different dimensions of interest and characteristics of the relationship between two datasets can be studied.

\subsection{Time scale v/s Spatial scale}

This method of averaging is produces results very similar to the 2-D Fourier transform. In case of a Fourier transform, a global average of the spectrum alone can be obtained. Using this method, we an obtain such spectrum over a region in space and a time interval, thus providing localization in both the domains.

$$
\hat{F}\left(s_{x}, s_{t}\right)=\sum_{x_{1}}^{x_{2}} \sum_{t_{1}}^{t_{2}}\left|w\left(s_{x}, s_{t}, x, t\right)\right|^{2}
$$

In the case, where $x_{1}, x_{2}, t_{1}$ and $t_{2}$ are chosen such that they span the entire dataset, differences are noticed in the spectra generated by the conventional Fourier transform and the 2-D wavelet method. [5] demonstrated the difference between Global Wavelet Spectra and the Conventional Fourier Spectra at low and high frequencies. [6] also noticed the problem of loss of frequency localization at high frequencies and time localization at low frequencies, and suggested that this could induce the difference between Global Wavelet Spectrum and Fourier spectrum. Sudden changes in data can also cause spectral smearing. These can however be jdentified in the time/space domain because of their short duration.

\section{STUDY OF NORTHWARD PROPAGATIONS - AN APPLICATION}

[7] first showed that poleward movement of organized cloud bands occurred over the Indian longitudes with a time period of approximately 45 days. [8] showed that similar phenomenon also occurs in the West Pacific. We analyzed this phenomenon using two-dimensional wavelets and obtained similar results.

The 2-D wavelet analysis was used to determine the space-time characteristics of propagations over a given longitude. 2-D latitude-time data segments were constructed from Daily Precipitation dataset $\left(1^{\circ} \times 1^{\circ}\right)$ from the GPCP database (1997 on wards). The data sets spanned from March 1st of a year to the 28th February of the next. The data from $80^{\circ} S$ to $80^{\circ} \mathrm{N}$ was used to provide sufficient number of points in the latitude domain to keep our region of interest well within the cone of influence. The time-averaged spectrum $\left(\hat{P}\left(s_{x}, x\right)\right)$ for the period from June-September were computed for the data sets.

\subsection{Dominant spatial scales}

When the analysis was performed at intervals of $10^{\circ}$ of longitude, it was observed that significant power occurs at $90^{\circ} \mathrm{E}$ and $150^{\circ} \mathrm{E}$ in June to September every year. Some power is also observed at the $70^{\circ} \mathrm{E}$ and $80^{\circ} \mathrm{E}$. This activity is at least 95\% significant (compared with an AR-1 model at 244 d.o.f ( Shanker and Nanjundiah (2004)) with a $32^{\circ}$ spatial scale and is observed just to the north of the equator (Fig. 2). This is a typical characteristic of Poleward propogations of monsoons in Bay of Bengal and the Pacific. Such characteristic features are not observed at other longitudes.

\subsection{Time-frequency analysis}

Space averaged scalogram $\left(\hat{Q}\left(s_{t}, t\right)\right)$ for the dominant $32^{\circ}$ scale was obtained over the region $\left(10^{\circ} \mathrm{N}-20^{\circ} \mathrm{N}\right), 30-45$ day cycles are observed in the scalograms for all the years (Fig. 3). Significant power is also observed in 15-day cycles in early June which corresponds to the onset of monsoons. Such 15-day cycles have been observed by [9]. The $32^{\circ}$ spatial scale dominates in all years, the time-evolution for this scale is different in all years indicating inter-annual variability of intra-seasonal variations.

Analysis of the temporal scales at $150^{\circ} \mathrm{E}$ shows a structure quite different from that over the Indian jongitudes. The 45-day scale scale seems to persist right from June to October. The 15-24 day cycles also seem to be significant in all the years. In addition to the information about the temporal scales, the 2.D wavelet method also provides us information about the spatial scales which the other methods fail to do.

\section{CONCLUSIONS}

2-D wavelet analysis is a very useful method in achieving time and space localized spectral information. The method clearly helps us identify various local features in the spectra. The method can be extended to multi-dimensional data sets. When used in combination with effective multi-dimensional local search algorithms, wavelet analysis can become a very powerful tool for identifying signature characteristics of wavelike phenomenon.

Analysis of the latitude-time data set shows that around $30^{\circ}$ spatial scale is prominent with a dominant temporal scale of 45 days in the region $10^{\circ} N$ to $20^{\circ} N$ over the Indian latitudes(maximum at $90^{\circ} \mathrm{E}$ ) and the pacific(maximum at $150^{\circ} \mathrm{E}$ ). These are the typical features of poleward propagations of the monsoons over the Indian longitudes and the pacific. Such prominent spatial scales are not seen over other longitudes. Moreover it was also observed that though the dominant spatial scales remain the same every season, significant inter-seasonal variability is observed in the time scales. 


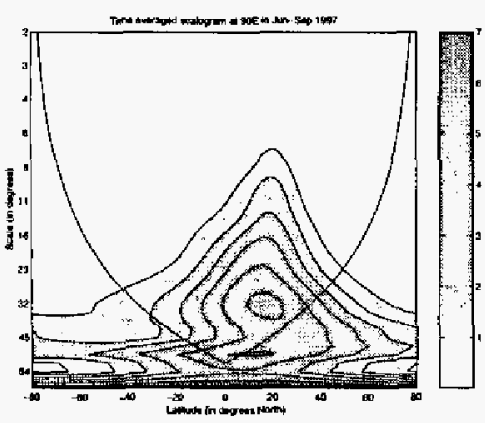

(a)

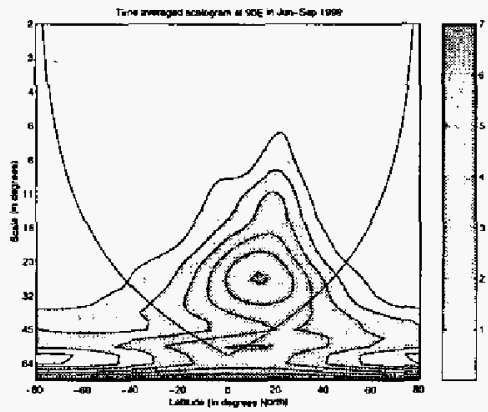

(b)

Fig. 2. The figure shows the time averaged scalogram for the period (a) Jun-Sep, 1997 and (b) Jun-Sep, 1999 of rainfall data spanning latitudes $80^{\circ} \mathrm{N}$ to $80^{\circ} \mathrm{S}$ and averaged over the region $85^{\circ} \mathrm{E}$ to $95^{\circ} \mathrm{E}$. The $32^{\circ}$ spatial scale is prominent in the period June-September of every year. Activity is strong in the region $10^{\circ} \mathrm{N}$ to $20^{\circ} \mathrm{N}$. The cone of influence (region affected by periodic wrapping of data) has also been shown.

Thus two-dimensional wavelet is a simple yet effective tool to obtain information on spatial and temporal scales and their localization in time and space, making it very effective in characterizing wave-like structures.

\section{REFERENCES}

[1] Yoshikazu Hayashi, "Space-Time spectral analysis using the Maximum Entropy Method," J. Meteor Soc. of Japan, vol. 55, pp. 415.420, 1977.

[2] C. Torrence and G. P. Compo, "A Practical Guide to Wavelet Analysis," Bull. Amer. Meteor. Soc., vol. 79, pp. 61-78, 1998.

[3] Guojun Gu and Chiding Zhang, "A spectrum analysis of synoptic-scale disturbances in the itcz;" J. Climate, vol. 14, pp. $2725-2739,2000$.

[4] Piyush Shanker A, and Ravi S. Nanjundiah, "Morlet wavelet analysis of tropical convection over space and

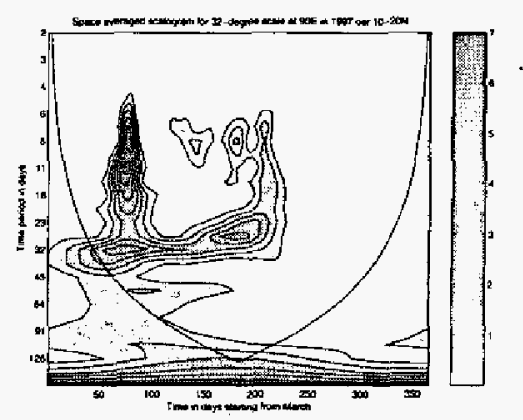

(a)

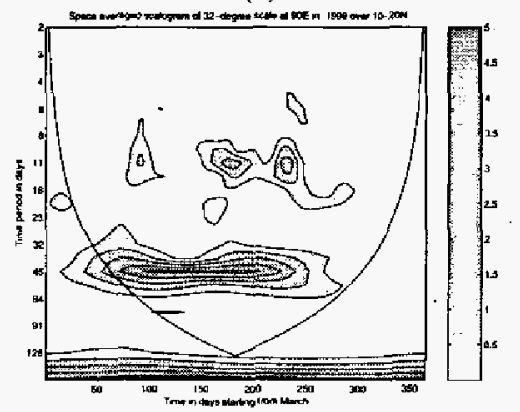

(b)

Fig. 3. The figure shows the space-averaged scalogram for a $32^{\circ}$ spatial scale over the longitudes $10^{\circ} \mathrm{N}$ to $20^{\circ}$ for the years (a) 1997 and (b) 1999 for the same data set as Fig. 2. The $30-45$ day temporal scale seems to prominent at this particular spatial scale. The cone of influence is also shown.

time: Study of poleward propagations of intertropical convergence zone (itcz)," Geophysical Research Letters, vol. 31, no. 2, 2004.

[5] V. Perrier, T. Philipovitch, and C. Basdevant, "Wavelet spectra compared to Fourier spectra," J. Math. Phys., vol. 36; pp. 1506-1519, 1995.

[6] B. Wang and Y. Wang, "Temporal Structure of the Souther Oscillation as revealed by waveform and wavelet analysis," J. Clinate, vol. 9, pp. 1586-1598, 1996.

[7] D. R. Sikka and Sulochana Gadgil, "On the maximum cloud zone and the ITCZ over Indian longitudes during the Southwest Monsoon," Mon. Wea. Rev., vol. 108, pp. 1840-1853, 1980.

[8] J. Srinivasan and G. L. Smith, "Meridonial migration of tropical convergence zones," J. Appl. Meteorology, vol. 35, pp. 1189-1202, 1996.

[9] T. N. Krishnamurthi and H. N. Bhalme, "Oscillations of a monsoon system. part i. observational aspects.," $J$. Atmos. Sci, vol. 33, pp. 1937-1954, 1976. 